\title{
Research on the Establishment of College Students' Leisure Oriented Health Physical Education Model
}

\author{
Linbao Zhang ${ }^{a}$, Haibin Xiao ${ }^{b}$ \\ School of PE, ShangRao Normal University, Jiangxi Shangrao 334000, China \\ a3235777167@qq.com, b594481921@qq.com
}

Keywords: college students, leisure orientation, health, Physical Education.

\begin{abstract}
This study through literature method, field survey method, observation method, interview method, logical reasoning and other research methods, on the recreation orientation in university students' physical health education process of role taking to explore, in the course of the study from the following aspects: the meaning of leisure oriented, leisure oriented characteristics, recreation orientation and physical education, college students leisure oriented physical education mode. The aim is to provide reference for the establishment and development of health physical education model in college physical education.
\end{abstract}

\section{Introduction}

Health education is a long time students education "grand opera", is also a school sports has been sticking to the main vein. Sports peers are in research, in the hope of better health education mode is put forward. Leisure directional movement is a natural and humanistic sports of double platform, implement and adhere to in the college students' health education, must bring important role for the effectiveness of the system of college health education, so the worthy of attention.

\section{The leisure directional meaning}

Leisure sport began in some developed countries in Europe and the United States more prevalent. After the founding of new China, our country already has a certain development of recreational sports. Import in our country market economy system since 1978, the national economy high speed development, people's living standard has improved greatly, so as to establish the concept of the initial size of leisure sports. The development of leisure sports and the same period of the social, economic and cultural development are closely related, a period of the more advanced the society, economy, culture, leisure sports will carry out the better. Along with our country economy enters a high-speed development period, the national life level gradually improve, leisure time gradually increase, people pay more and more attention to the development of its own, especially the spiritual demand is more and more people's attention. Functions and characteristics of leisure sports itself has, to some extent to meet the spiritual needs of people, because of its content, flexible and varied in form, by more and more people like it.

Orientation should be able to understand it: it is a kind of goal orientation, is a kind of pursuit, is also a kind of transcendence. If the use of sports psychology achievement goal orientation and learning goal orientation in the ranks, thought to be more kind of goal orientation. Directional movement according to the different classification standard has many forms, this research defines: leisure orientation is directional (a type of directional cross-country) on foot, is with the aid of ones, and maps, in all kinds of natural environment and human environment to complete the intended target activities by way of aerobic exercise. Leisure foot directional movement can give full play to the brain governs function, body in harmony and reaction in the brain, promote harmonious and healthy development of the organism. 


\section{Leisure directional characteristics}

Leisure directional features are various: as for the movement of their own way, has its natural attributes or natural characteristics; In terms of the sport as a social activity, has its social attributes, namely social characteristics; Recreational orientation is a sport, it and other projects, is a kind of physical activity. Took the form of human movement as the main characteristics, so there are human body dynamic characteristics; At the same time, leisure orientation is special in it was conducted with the aid of ones, map of intelligence and physical integration of leisure sports, therefore has the intelligence and physical characteristics. Have the characteristics of directional movement leisure orientation, also has its own characteristics. The specific movement characteristics as follows:

\subsection{Intelligent}

Leisure orientation is a combination of physical and intellectual movement. In terms of intelligence, the first to have geography, surveying and mapping science, military topography and other related knowledge, and ability to use the knowledge.

\subsection{The natural environment}

Leisure directional movement is in the wild, forests, mountains, park, scenic spot and so on of the natural environment. This is it with all kinds of sports in sports venues for a significant difference. Recreational orientation activities and the venue environment particularity is to determine the sport more able to attract people to participate in one of the important factors.

\subsection{Interest sex}

Leisure directional movement environment, activities and methods, etc, can arouse people's interest, more fun of play activity, mining the greatest positive emotions of potential, enhance people to participate in the initiative and enthusiasm.

\subsection{The game entertainment}

The leisure directional movement gameplay is very obvious. From the early stages of its development, Sweden's scout \"treasure huntl", itself is a kind of game, until the modern variety of directional game, is still a great game.

\subsection{The leisure athletics}

Recreational orientation can undertake all types of games, the competitive characteristics also have highlighted. Leisure orientation and general directional sports has the difference is that people first in natural light, enjoy the directional pursuit of competition, is the competition, the pursuit of sex so that reach the level of them.

\subsection{Mass}

Recreational orientation of popularization rate is very wide, its for object is very wide, both male and female, young and old, can become the sport of the participants and enthusiasts. According to foreign reports, directional movement competition, the youngest of 8 years old, most elderly people at the age of 80 . So it can be said that leisure directional movement is a popular sport.

\subsection{Practical}

Recreational orientation can be an important part of People's Daily life way, in the aspect of promoting people's health and happiness will have very good leading and concrete operational, at the same time, leisure orientation in school sports purposes comply with the principle of physical education in the process of implementation and departments at all levels of file spirit, at the same time fit students needs the innovation of the physical education mode reform.

\section{Students' physique observation situation analysis}

According to the national student physical health standard "for a university student physique sampling test. Basic situation and the test items include: gender, age, height, weight, vital capacity in succession, crook proneness, sit-ups (female), standing long jump, pull-ups (male), 50 meters, 800 meters (female), $1000 \mathrm{~m}$ (male). In order to guarantee the uniformity, accuracy and reliability of the data, all the participants in the tester are unified training before physical test. 
Physical test score results show , the college student's physical development in except boys pull-ups are shown to pass the above, but unevenly. The power of the college students' quality and level of age should have appeared deviation phenomenon should arouse our attention. The development of students physical fitness test and the analysis of test results can be scientifically guiding the future focus direction in daily physical activity, effectively develop fitness exercise prescription. From observation test results can be clear is to pay attention to the muscle strength of boys, girls muscular endurance and flexibility of exercise to improve their physical quality; In addition to pay attention to college students of the imbalance in the fitness testing, to formulate some focus on strength, stamina, flexibility, coordination and so on aspects of the exercise scheme in school physical education and extracurricular sports activities. Body movement, in short, through various forms and physical tests, scientific and reasonable selection of sports content, innovation of physical exercise methods, etc., let the students really enjoy "dividend" sports, promote the visual, hearing, touch, sense of equilibrium, spatial perception, motion perception and health physical quality coordinated development, such as for physical development, psychology and intelligence development lay a solid foundation, so that the students can fully develop mind and body.

\section{The leisure orientation relationship with physical education}

Leisure orientation is a kind of for the purpose of leisure sports activities, is a kind of body and mind of perfect combination of sports. It not only can relieve stress, relax too much tension, more can make public individual character, pursuit of taste and interest. Directional is a healthy sport leisure and fashion combination of spiritual and cultural pursuit of a way of leisure. In the event, people can leisure attitude, leisure participation way to engage in the activities, is an essential part of people "in fitness project" an important part. It also will be an important part of college campus sports culture, bring help for college students' healthy growth.

As the example to define physical concept refers to the human body on the basis of genetic and acquired displayed on the function and form of the intrinsic characteristics of relative stability. Is a medical term. Physical quality can affect the individual's response to external stimuli and adapt. As early as the word "physical education" is Mr Xu Yingchao is put forward in 1979, which promoted the development of our country's sports teaching scientific. At the same time, many scholars advocate the "sports (movement) lesson" as "physical education" to better reflect the sports education value. Physical education it is necessary to define the problem of two aspects, one is to make students form a kind of consciousness, 2 it is to let the students take the initiative to participate in specific practice of enhanced physique. This is not just, Shouting slogans more educators need to continue to think and study, to work out more effective ways and means, so that to achieve the goal of physical education.

Leisure directional relationship between physical education establish think of it this way: the difference is recreational orientation, is a kind of sport is a kind of activity, match with the concept of "movement", indicates that leisure directional movement in the process of changing people can play an important role, especially health and fitness. Physical education is one of the school sports education pattern, is to improve college students' physical fitness methods and strategies adopted by the arrangement and plan. For physical education integration pattern includes leisure directed the content system, recreational orientation for physical education mode to broaden the content strategy more effectively.

\section{Directional health leisure physical education model strategy}

\section{1 efforts to build and create directional environmental health education of college students leisure atmosphere.}

University campus environment is one of the important part of the "physical environment". Leisure directional environment can not only affect the effect of a school, training talents, the important thing is that can bring vitality to the school. Health development is to cultivate students' main guiding direction, and directional as leisure health interventions are particularly important. Leisure directional 
environment can through the school of publicity column, modern information platform, held colorful bright and happy recreational orientation activities and competitions, founded by school association of directional movement, leisure and social sports organizations to establish collaboration system, etc. Not only to the school sports culture, more important can stimulate the student to understand the leisure directional movement, stimulates the student to participate in the leisure the motive of directional movement, promote the development of college students' health.

6.2 college students' leisure targeted health education to break through the traditional theory of single form of body movement, from technology to technology should be diversified, establish scientific and effective pattern of leisure directional movement.

Leisure science including the correctness of the movements of directional movement and the exercise methods and principles, corresponding to the leisure directional exercise effective threshold, sports nutrition and health care, reasonable physical exercise plan, the right mindset, etc. Leisure directional education mode should be follow the scientific, on the basis of aerobic leisure directional movement as the main form, reasonable arrangement of choosing leisure directional movement project, is set leisure directional movement rhythm of the interval, effective planning leisure prime-time directional movement at a time.

\section{3 establish recreational orientation from thinking training of standard system health education model.}

Training is in his sports activities as the carrier, in the psychological cognition as the breakthrough point, by means of organizational behavior, and rise to the theory of management; It is cultivate the psychological quality, stimulate individual potential and to improve survival skills, strengthen the team spirit of comprehensive activity. leisure directional health education should fully absorb the outward bound training experience, first to share again, after summarizing development pattern. Let college students through a recreational orientation activities, achieve the goal of sustainable comprehension and education.

\section{4 directional health leisure physical education model, to be highly qualified teaching staff.}

For leisure orientation in the process of physical education in colleges and universities should constantly optimize teaching staff, we regularly organize recreational orientation will teachers' teaching and research, regularly send relevant teachers to participate in domestic and international academic exchange and the related training of directional movement, high level base experts regularly invited to the school to give lectures, etc.

\section{5 recreational orientation in colleges, alliance system can implement regional colleges and universities.}

A college leisure directional alliance website, will be complementary advantage resources for communication; Field performance regularly sent teams, exchanging experience, innovative leisure directional education new mode; Recreational orientation from establishing modern communication platform between teachers and communicate with each other all sorts of orientation to carry out the way and the latest recreational orientation information dynamic.

\section{6 to establish a scientific and effective directional health leisure physical education evaluation} mechanism.

Directional health education evaluation mechanism is related to the recreational development of directional movement, it should be different from general sports project evaluation, the main should learn from the students' well-being index, physical fitness, attitude and behavior, solidarity and cooperation, to explore the innovation of refinement measure index system. Evaluation mode can take mutual between team and group self-evaluation, mutual between groups, the teacher reviews, evaluation method, etc.

6.7 project into more, the continuation of recreational orientation of physical education activity.

Interest is the best teacher, novelty is the guarantee of student motivation. Recreational orientation, therefore, should constantly develop and change the content of the system, based on the nature of recreational orientation, move other projects from recreational orientation, the content system of 
leisure orientation more rich. Such as cross-country walk, parkour, rainbow run into modern popular sports leisure orientation should be a very good choice.

\section{Conclusion}

College students' health education pattern is in "physical education" and "the concept of sanki sports" touch the university sports teaching dominant mode. College students health education should be to strengthen students' physical quality education on the basis of deepening reform, the sports knowledge education, sports ability education, psychological education and the sports quality education as the main content of college physical education teaching. The current college students' health education will focus on deepening the reform of physical education teaching mode, improve the quality of classroom education inside and outside, inside and outside education mode, change the sports course students' health education pattern reform ideology construction. Leisure directional physical education mode is derivative of the reform of school physical education at the university of innovation. Its survival and development must leave the school sports law of development, but its existence is not regular sports activities project change. Recreational orientation should stick to go the way of their own characteristics, create diversified recreational orientation "brand".

\section{References}

[1] Zhang Xiaowei. Directional cross-country [M]. Star map publishing house, 2013

[2] TaoYuPing Peng Fudong, etc. The school training [M]. People's sport publishing house, 2008

[3] in. Restriction directional cross-country course conducted by factor analysis [D]. Shandong normal university, 2013

[4] Xu Jiahui, selected, etc. To build "directional cross-country" theory and practice of physical education curriculum mode study [J]. Journal of guangzhou sports institute, 2008, 28 (4): 44-47 (51)

[5] $\mathrm{Xu}$ An. Directional cross-country university of jiangxi province ordinary university sports curriculum present situation research [D]. Jiangxi normal university, 2013 